\title{
Self-starting Simulation of a Hypersonic Inlet with Variable Free Stream Condition
}

\author{
J. $\mathrm{Wu}^{1,2 \dagger}$, A. Y. Yu ${ }^{2}$, Y. Y. $\mathrm{He}^{2}$ and J. L. $\mathrm{Le}^{2}$ \\ ${ }^{1}$ School of Power and Energy, Northwestern Polytechnical University, Xi'an, Shanxi,710072, China \\ ${ }^{2}$ China Aerodynamic Research and Development Center, Mianyang, Sichuan,621000, China
}

†Corresponding Author Email: wujie240163@126.com

(Received January 27, 2020; accepted April 17, 2020)

\begin{abstract}
The flow structure transition of a hypersonic inlet from unstart to start during accelerating trajectory is studied by numerical simulation. The results of pressure distribution along the inlet wall, mass flow rate, total pressure recovery coefficient and aerodynamic forces simulated by the quasi-steady and unsteady two dimensional and three-dimensional quasi-steady methods are compared. Analysis indicates that the twodimensional quasi-steady and unsteady simulations can get the consistent inlet self-starting Mach number, and unsteady simulation can capture the periodical change of aerodynamic forces while this phenomena is not found in quasi-steady simulation. Due to the consistency between the quasi-steady and unsteady in selfstarting Mach number in our strategy, for the self-starting process of hypersonic inlet with variable free stream condition, three-dimensional quasi-steady method can be used to approximate the real self-starting process during the acceleration of the aircraft climb, which can greatly save the computational time and improve simulation efficiency.
\end{abstract}

Keywords: Hypersonic inlet; Self-starting; Variable free stream condition; Unsteady.

\section{NOMENCLATURE}

$\begin{array}{ll}C d & \text { drag coefficient } \\ C l & \text { lift coefficient } \\ C p & \text { pressure coefficient } \\ M a & \text { Mach number } \\ P & \text { inflow pressure }\end{array}$

\section{INTRODUCTION}

The primary purpose of a inlet of scramjet is to capture sufficient air, provide efficient shock compression, produce near-uniform inflow to the combustor, generate low drag, and provide these characteristics over a wide operating envelope. Starting a hypersonic inlet can be a very complex process that is influenced by a variety of aerodynamic phenomena, such as the flight conditions (i.e. angle of attack, Mach number, pressure of freestream) and disturbance of the combustor (Chang et al. 2008). Inlet starting is defined as the ingestion of normal shock trains from the front of the inlet through the inlet throat to a point downstream of the throat. It can be a very unstable condition, and the slightest perturbation can cause the normal shock trains to be ejected from

$\begin{array}{ll}t & \text { time } \\ \gamma & \text { specific heat } \\ \sigma & \text { total pressure recovery cofficient } \\ \Phi & \text { mass }\end{array}$

$\Phi$ mass flow rate the inlet (i.e., inlet unstart) (Heiser et al. 1994). Experiences show that the undesired unstart phenomenon may lead to violent shock system oscillations, prominent pressure fluctuations, and abrupt performance reductions which result in the substantial engine thrust loss and even the combustor flameout (McClinton et al. 1999; Tan et al. 2007). Due to the immaturity of design methods for hypersonic inlet, the inaccuracy of CFD (computational fluid dynamic) tools for hypersonic flow, and the inconsistency of ground simulated condition with flight condition, the unstart state is inevitable during the flight envelope. Especially, along the accelerating trajectory of vehicle, flight Mach number and other parameters are continuously changing, which is a typical unsteady flow in the inlet. At this stage, with the flight Mach number increased, inlet will transit from unstart to 
start (self-starting process).

Due to the complexity of the flows, inherent limitations of related ground-based and expensive flight experiments, numerical simulation is attractive for the analysis and understand the flow mechanism to gain further insight at this stage and has been applied for inlet unstart simulations (Tahhir et al. 2003; Sebastian et al. 2011; Jiao et al. 2015; Logan et al. 2018). As we all know, although CFD can solve many problems that can't be solved by hand or wind tunnel before, completely direct numerical simulation(DNS) is not practical now, even large-eddy simulation(LES) can not be applied in actual industrial hypersonic flow problems now. For many design departments, it is awe-inspiring to solve unsteady Reynolds average Navier-Stokes (URANS) equations for the actual industrial configuration. There are many literatures that study the factors that affect the start/unstart of hypersonic inlet (Hsieh et al. 1984; Mayer et al. 1994; Kopasakis et al. 2012; Grainger et al. 2012), but few have studied self-starting process with variable free stream condition. If we want to understand the detailed flow mechanism of the hypersonic inlet self-starting process during variable inflow conditions, and also meet the efficiency requirements so that the design department can quickly evaluate and iteratively optimize the configuration, we need to develop a new numerical simulation strategy.

In this paper, quasi-steady and unsteady numerical simulation tools are used to analyze hypersonic inlet self-starting capability when vehicle flies in a variable inflow condition. Compared to the traditional steady method which usually simulates in a fixed free stream condition, we simulated unsteady phenomenon in a variable inflow condition which is more realistic. The inlet starting Mach number range is determined through the quasi-steady simulation firstly. By comparing the two-dimensional and three-dimensional quasisteady simulation results, it is indicated that the flow field changing trend is consistent for twodimensional and three-dimensional inlet simulation before and after inlet start. Finally, the flow structure changing during the inlet unstart to start process are qualitatively researched by twodimensional unsteady simulation.

The organization of this paper is as follows. Section (1) is some background knowledge. Section (2) is a brief introduction about inlet model and the numerical method used; the main body is section (3) which contains three subsection. In (3.1) the authors mainly study two-dimensional (2D) quasisteady flow in order to describe typical flow characteristic and to get the inlet self-starting Mach number range to serve two dimensional unsteady simulation. In (3.2) the three-dimensional (3D) quasi-steady simulation is implemented in order to find the difference from two dimension. Section (3.3) illustrates differences between quasi-steady and unsteady results and concluding remarks are drawn in the final section.

\section{MODEL AND NUMERICAL METHOD}

\subsection{Inlet Model}

The present model is a two-dimensional planar mixed-compression inlet with overall contraction ratio (CR) 7.4 and internal CR 1.5. The designed Mach number is 6.0 with four compression corners which consist the external compression surface with total deflection angle 20.7 degree. The internal compression surface is composed of three corners of the inner wall surface of the cowl, and an arc is used for the transition between the internal and external compression surface. The total length from the leading of inlet to the exit of isolator is about $50 \mathrm{H}(\mathrm{H}$ is the throat height) and the height of isolator is $8.3 \mathrm{H}$. Figure 1 shows the sketch of the model. At the design point (Fig.2), four oblique shock waves formed by the external compression surface intersect at the lip of cowl. But the capture air mass flow rate of the inlet will decrease rapidly because of the strong shock-wave/boundary-layer interaction under off-design point(Fig.3), resulting in the dreadful shock wave drag and spillage drag, the great nose-up pith moment, the deterioration of the combustion efficiency and even the unstart of the inlet. It is the focus of this paper for the transition from unstart to start of the inlet because of accelerating trajectory of vehicle.

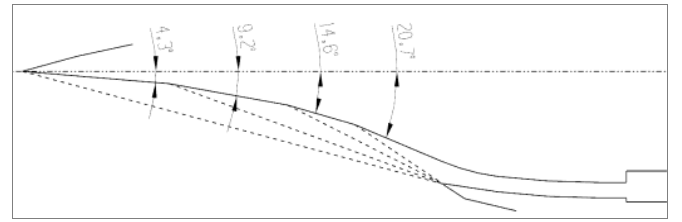

Fig. 1. Geometric sketch of the model.

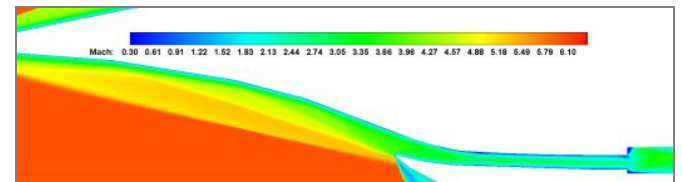

Fig. 2. Mach number contours (Mach 6).

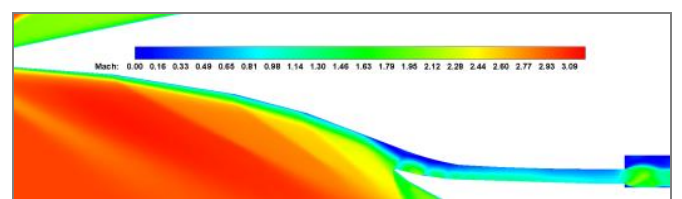

Fig. 3. Mach number contours (Mach 3).

\subsection{Grid and Numerical Method}

The two-dimensional computational grid total number is 185,800 and the three-dimensional one is 4.84 million. All the grids are refined near the wall to the $0.001 \mathrm{~mm}$, as shown in Figs.4-5. All two and three dimensional cases are solved by code AHL3D (Airbreathing Hypersonic Laboratory), which is a threedimensional, parallel, multi-block structured grid with chemical reaction solver in the frame of finite 
volume method developed by China Aerodynamic Research and Development Center (CARDC). It can solve 2D/3D/axisymmetric, steady/unsteady, Euler/laminar/turbulent and chemical flow with a variety of numerical schemes and turbulent models (Wu et al. 2018). All simulations solve Navier-Stokes equations and the air is calorically perfect gas with constant ratio of specific heat. For spatial discretization, cell interface quadrature point value is firstly reconstructed by MUSCL scheme (Leer, 1979) with Albada limiter and then AUSMPW ${ }^{+}$scheme (Kim et al. 2001) is used in cell interface to get inviscid flux. The second-order accuracy is got by Gaussian formula around cell interface for viscid flux. In order to simulate unsteady flow, dual-time step method (Jameson, 1991) is used for temporal discretization. This paper chooses two-equation eddyviscosity shear-stress transport model (SST) (Menter, 1994) for turbulence computation because it led to higher accuracy of the simulation of the whole flow field by treating the high-speed flow in the main stream with k-epsilon model and treating the low velocity flow in the near-wall region with the standard k-omega model. The final linear equations are solved by LU-SGS scheme (Yoon et al. 1988). The wall boundary condition is no-slip and adiabatic. The other boundary conditions are far field, symmetry, pressure outlet. For unsteady variable inflow condition simulation, we linearize the Mach number and the inflow pressure with time by analyzing trajectory parameter. For detailed linearization formula and discussion, please refer to the section (3.3).

The selected Mach number range along accelerating trajectory of the vehicle is from Mach 3.0 to Mach 5.0. The inlet does not start below Mach 3.0 and completely starts above Mach 5.0. Mach 3.0 incoming flow condition of the flow field is calculated by steady results for quasi-steady. Unsteady simulations use the steady flow field results as the initial flow field and quasi-steady simulations use the previous state of the flow field as the initial flow field.

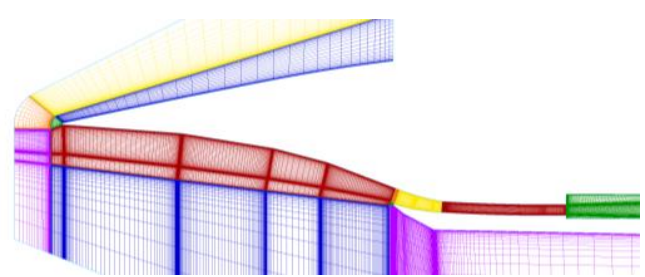

Fig. 4. Two-dimensional computational grid.

\section{RESULTS AND DISCUSSION}

\subsection{Two-Dimensional Simulations}

Quasi-Steady

Figure 6 shows the pressure coefficient distribution along the inlet wall from the leading edge to the outlet of different inflow Mach numbers. It can be seen from the figure that the pressure distribution is divided into three groups. The first group is from
Mach 3.0 to 4.05 , the second group corresponds to the state from Mach 4.1 to 4.35 and the third group is from Mach 4.0 to 4.6. The pressure coefficient distribution of the first group suddenly changes to that of the second group from Mach 4.0 to 4.1. In order to confirm that this process is indeed a sudden change rather than because the Mach number's change of 0.1 is too large to capture the leap, steady-state flow convergence results of Mach 4.0 are used as initial field to calculate the flow field of Mach 4.05. Figure 3 shows that Mach 4.05 is in the same group as Mach 4.0 and there is no intermediate state between them. This range is also calculated using Mach 4.35 to prove that from Mach 4.3 to 4.4 actually there is a sudden pressure change.

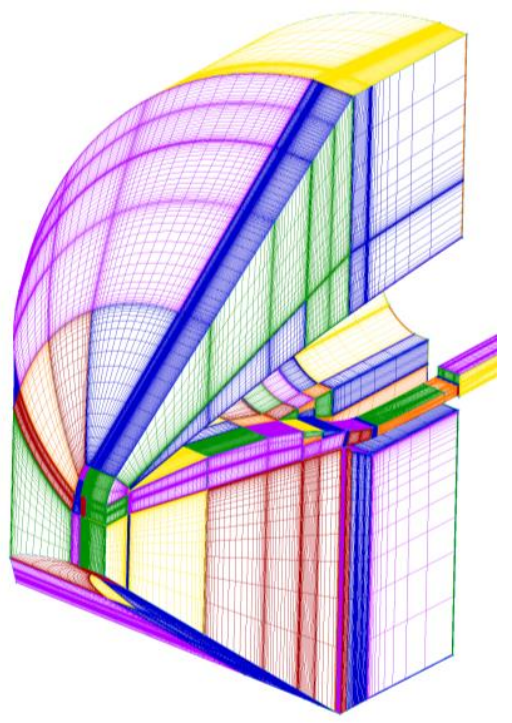

Fig. 5. Three-dimensional computational grid.

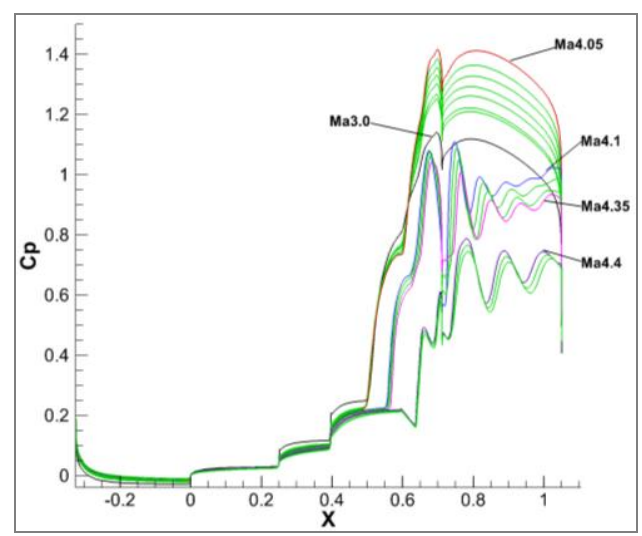

Fig. 6. Pressure Coefficient distribution of twodimensional quasi-steady simulation.

In Fig.6, all states of the first group are unstart, and the pressure distribution after about $\mathrm{x}=0.6 \mathrm{~m}$ from inflow Mach 3 to Mach 4 increases with Mach number. That is because the inflow Mach number and the kinetic energy continues to increase, which causes the pressure rises with Mach number. It can be seen from the pressure coefficient distribution of the first 
and the second groups, three stages of external compression surface produce three oblique shock waves making pressure increased three times. The pressure of the first group near the place of $x=0.5 \mathrm{~m}$ has a big increase, starting from the shoulder point where unstart inlet generates a large separation bubble. An oblique shock wave induced by the front of separation bubble makes the big pressure increase and the inlet flow field does not start at this time. Another phenomenon is the interference of lip detached shock wave and the separation bubble (seen from the two marked locations in Fig. 7).

When the incoming flow Mach number increases to 4.1, from the Mach contours in Fig.8, it can be seen that the strong shock wave and separation bubble interference disappears, the separation bubble induced by oblique shock wave and lip shock wave interaction is clearly visible. Compared to the pressure distribution of first group, the separation bubble becomes smaller and moves forward, leading separation bubbles induced by oblique shock wave move forward too, resulting in the pressure rise of group two occur in the $\mathrm{x}=0.55 \mathrm{~m}$ nearby. The flow structure corresponding to the second group may be defined as transition state from unstart to start. The typical characteristics of the transition state is though large separation bubbles exist at shoulder, the lip detached shock is sucked in the inlet convergent section and the separation bubble induces oblique shock interaction with front lip detached shockwave.

With further increase in Mach number, the interaction of lip detached shock wave and oblique shock generated by compression surface is further increased. The inlet shoulder separation bubble becomes smaller (Fig. 9). When the stream Mach number increases to 4.4 , the separation bubble disappears, and the inlet is started, as shown in Fig. 10. In the period of inlet start above Mach number 4.6 , with the Mach number increasing, the pressure peak position moves forward continuously. This is due to the increase of Mach number causes isolator shock train moves forward constantly.

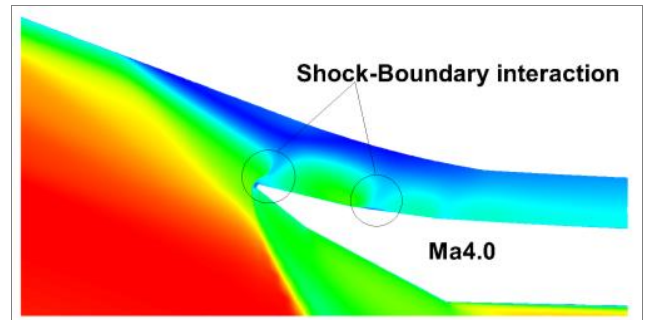

Fig. 7. Mach number contours (Ma4.0).

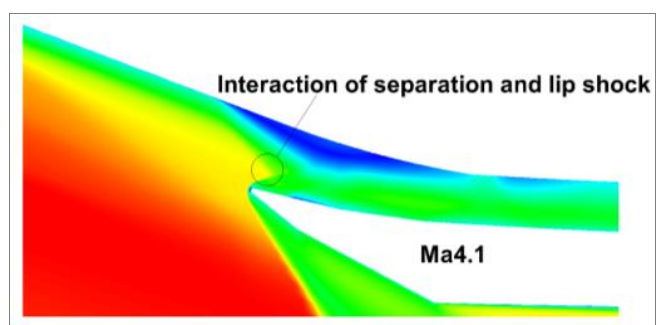

Fig. 8. Mach number contours (Ma4.1).

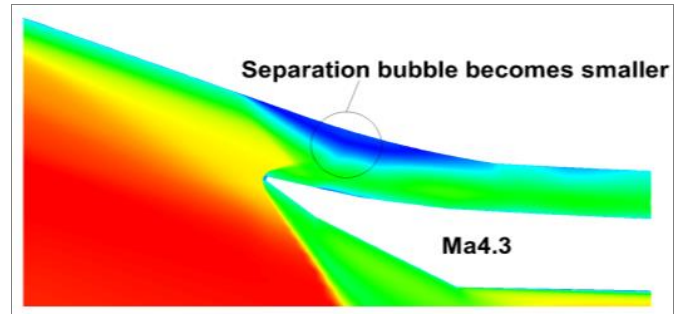

Fig. 9. Mach number contours (Ma4.3)

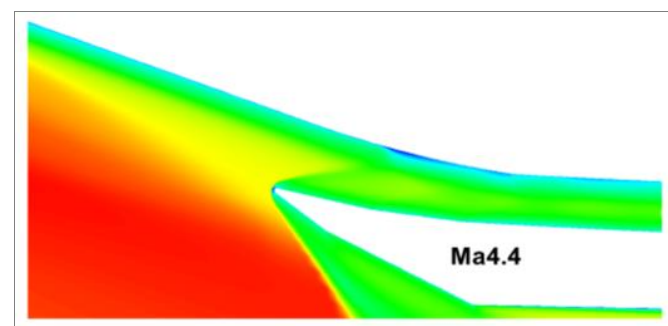

Fig. 10. Mach number contours (Ma4.4).

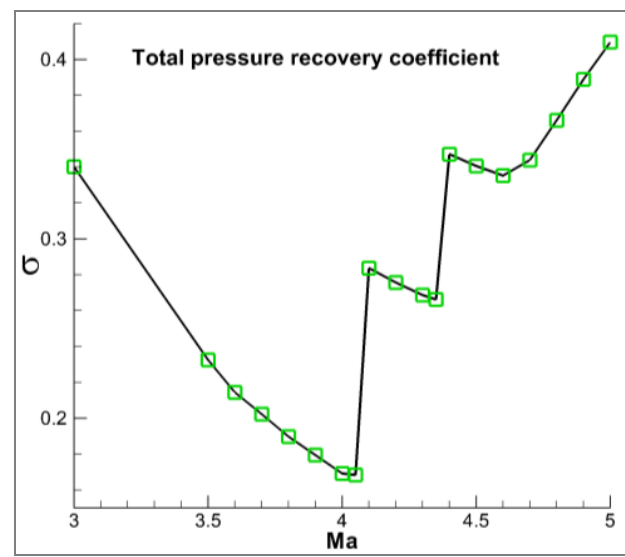

Fig. 11. Total pressure recovery coefficient.

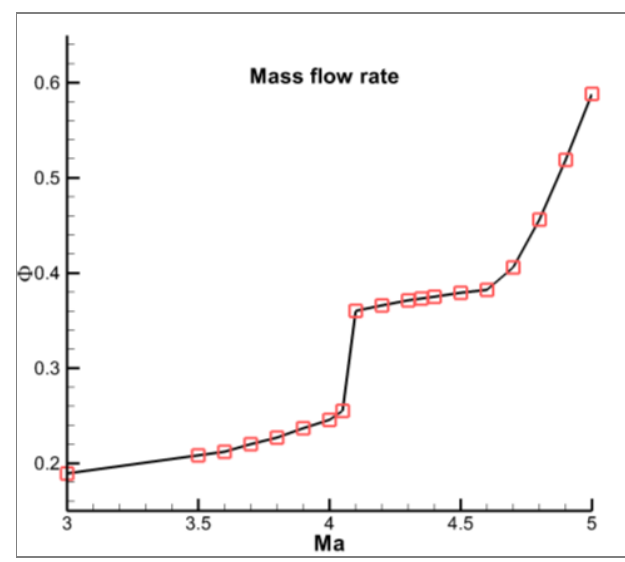

Fig. 12. Mass flow rate.

Total pressure recovery coefficient and mass flow rate are shown in Figs. 11-12. From the total pressure recovery coefficient graph, it can be seen the inlet is in completely unstart interval (inflow Mach number less than 4.0). The greater the inflow Mach number, the stronger the shock, results in a greater total pressure loss. On the other hand, the 
flow cannot be fully captured into the throat at this time, combined with a serious shoulder separation leading to the formation of clogged pneumatic throat, blocking airflow constantly accumulated before the throat. Both reasons will cause energy loss, making the total pressure recovery coefficient decrease and pressure increase. For inflow Mach number less than 4.0, the oblique shock wave produced by wedge surface detaches away from the cowl lip, resulting in a great spillage, and at this time the oblique shock pressure rise is relatively large, so the pressure of the first group is larger than the second group. When inflow Mach number increases to 4.1 , as the lip shock has finally been swallowed into the inlet, the spillage is reduced and mass flow rate is increased significantly. From unstart to transition state, and from transition state to start, the inlet drag (Fig. 13) drops twice and is consistent to the sudden changes of pressure distribution and the total pressure recovery coefficient, indicating that the inlet's drag greatly deceases from inlet unstart to start. The lift (Fig. 14) only jumps sharply once between Mach 4.0 and 4.1.

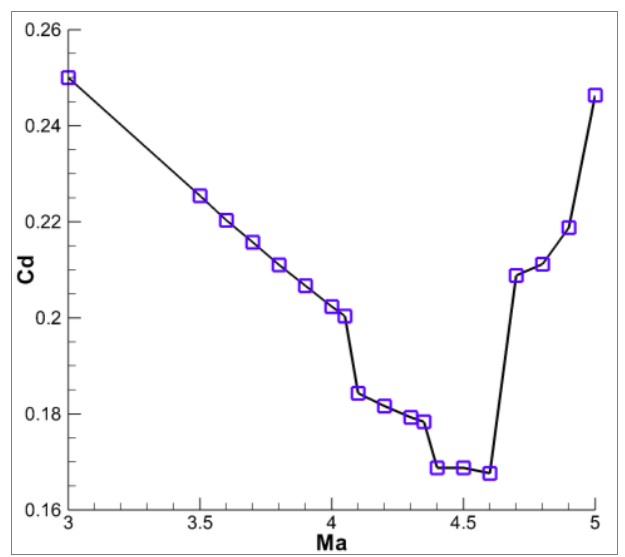

Fig. 13. Drag coefficient of inlet.

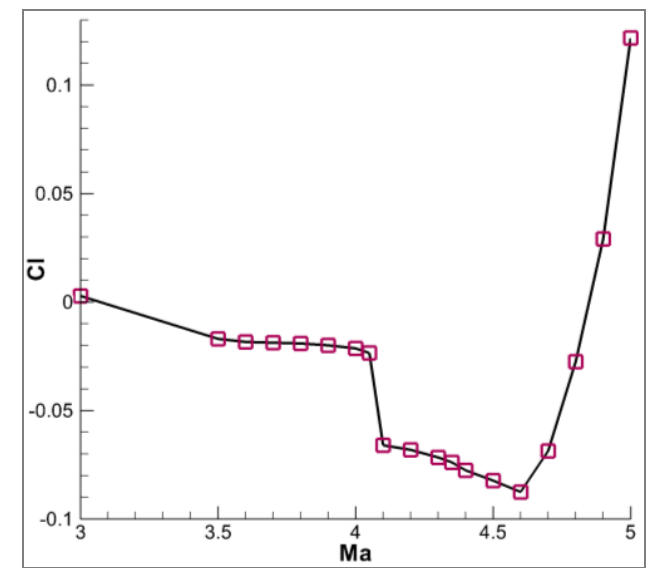

Fig. 14. Lift coefficient of inlet.

Remark I: When the vehicle is climbing and the inflow Mach number is small, the scramjet inlet is unstart. The typical flow characteristics are that there is large separation bubble on the inlet shoulder and this bubble induces a oblique shock which interacts with the lip bow shock in front of cowl. In the internal compression section, there is a aerodynamic throat which blocks airflow and the loss of total pressure and mass flux is very large. When the inflow Mach number increases to a certain value, the lip bow shock was swallowed into internal compression section and the shock structure of flow field changed. Corresponding to this change, mass flux and total pressure recovery coefficient has a sudden increase. The flow field is a transitional state for inlet starting process after that. When the inflow Mach number continues to increase, the separated bubble becomes smaller and smaller until it completely disappears. The total pressure recovery coefficient has another big increase at a certain Mach number and after that the inlet is completely started.

\subsection{Three-dimensional Simulations}

Three-dimensional quasi-steady simulation starts gradually from Mach 3.0 to 4.1. Figure 15 shows the pressure distribution along the inlet wall, and Figs. 16-17 are the comparison of two-dimensional and three-dimensional quasi-steady simulating results of pressure recovery coefficient and mass flow rate. It can be seen from the pressure coefficient distribution (Fig. 15), the inlet does not start at Mach 3.9. But when incoming Mach number increases to 4.0 the inlet becomes started, indicating that three-dimensional quasi-steady simulating inlet start point is between Mach 3.9 and 4.0.

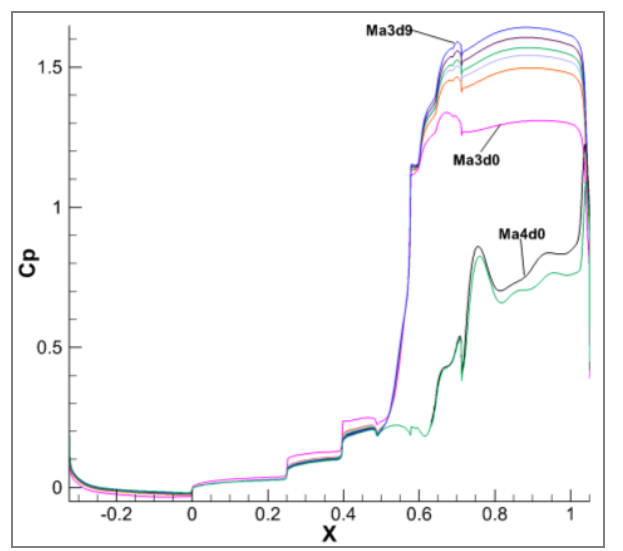

Fig. 15. Pressure coefficient distribution of threedimensional quasi-steady simulation.

Some conclusions can be made from the comparative analysis of Fig. 16 to Fig. 17. Firstly, regardless of two dimensional or three dimensional quasi-steady method, the inlet transits from unstart in low Mach number to start in high inflow Mach number. At some points, there is a sudden change in pressure distribution along the wall, total pressure recovery and mass flux coefficient. But the number of sudden changes and the change points are different. At the same Mach number, when inlet is unstart, the value of total pressure recovery and mass flux coefficient is different. In fact, we can draw a general rule: 
Regardless of the two dimensional or three dimensional simulation, the law of the air flow during the self-starting process of the inlet is similar from the perspective of the change of the flow field, but some physical quantities have different values from the quantitative point of view at the same Mach number.

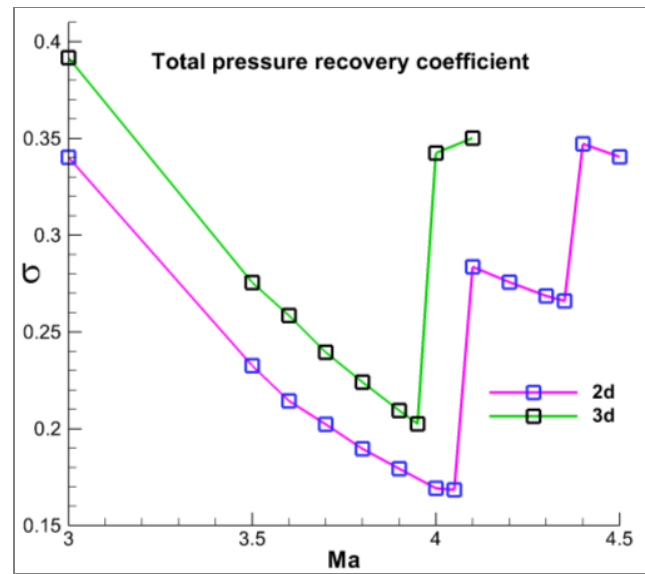

Fig. 16. Total pressure recovery coefficient of two-dimensional and three-dimensional quasisteady simulations.

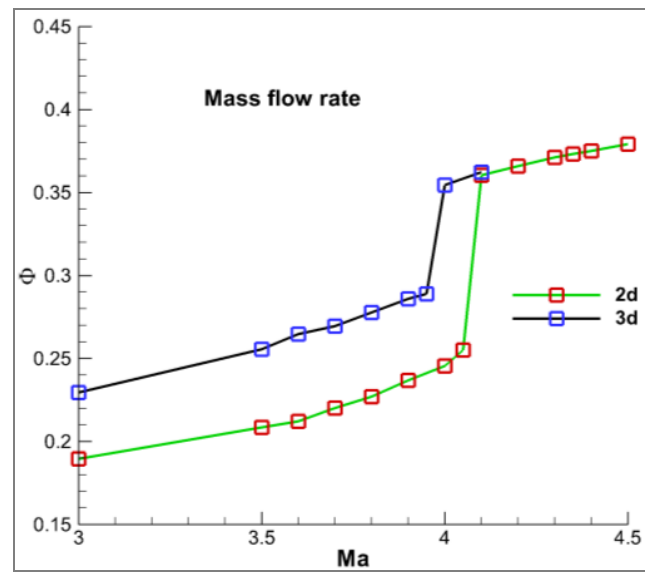

Fig. 17. Mass flow rate of two-dimensional and three-dimensional quasi-steady simulations.

Remark II: Why there are so many similarities and differences between two dimensional and three dimensional simulation? The author thought that the similarity represents the basic flow law of the airflow during the self-starting process of scramjet inlet. This process involves a series of changes in the flow field structure. There are separation bubbles on the shoulder of the inlet and spillage oblique shock induced by the bubbles, which is a typical flow field structure for unstart. With the inflow Mach number increasing to some point, the lip bow shock will be swallowed into internal compression section and the separation bubbles will become smaller to smaller until it vanishes completely. The difference between two dimensional and three dimensional is due to the three-dimensional lateral spillage. The state of inlet unstart is essentially a congestion of the air flow due to the pneumatic throat. The excessive airflow can flow away in the lateral direction in three dimensional configuration. The lateral spillage not only effect mass flux coefficient, but also effect self-starting Mach number. It is due to lateral spillage that it is easier to start the inlet by three dimensional simulation.

\subsection{Two-Dimensional Simulations}

Unsteady

The self-starting inlet Mach number is between 4.3 and 4.4 for two-dimensional quasi-steady simulation, 3.9 to 4.0 for three-dimensional quasisteady simulation. In order to accurately realize the parameter and flow field changes in the process of inlet unstart to start, unsteady simulation is necessary.

Two Mach number ranges of Mach 3.9 to 4.4 (Figs. 18-21) and Mach 4.3 to 4.5(Figs. 22-24) are simulated. We set a constant static temperature $(216.58 \mathrm{~K})$ and attack angle $\left(-6.3^{\circ}\right)$ through detailed analysis of trajectory data in these ranges. By simple linear data fitting, we set $\mathrm{Ma}=3.9+0.129456 \mathrm{t}, \mathrm{P}=9419.89-559.1772 \mathrm{t}$ for Mach 3.9 to 4.4 unsteady simulation, and $\mathrm{Ma}=4.3+0.13673 \mathrm{t}, \mathrm{P}=7612.73-465.89 \mathrm{t}$ for Mach 4.3 to 4.5 with physical time step $1.0 \mathrm{E}-06$ (s).

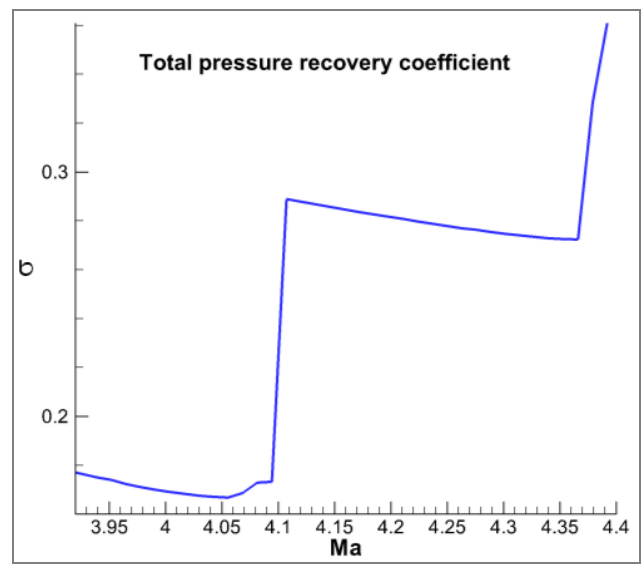

Fig. 18. Total pressure recovery coefficient of unsteady simulation (Mach3.9 to 4.4).

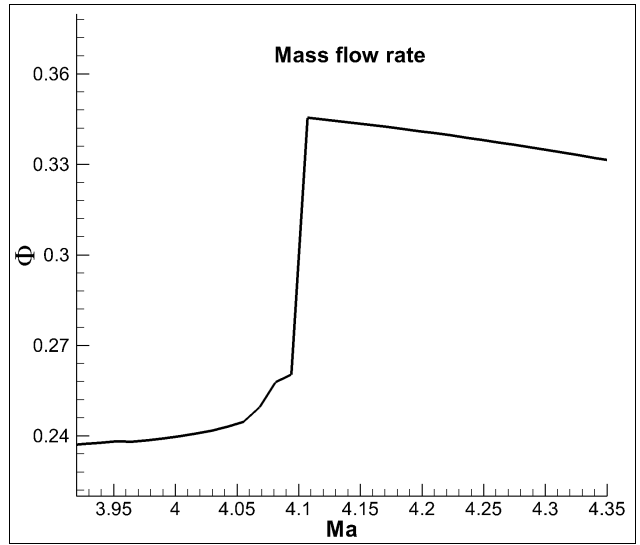

Fig. 19. Mass flow rate of unsteady simulation (Mach3.9 to 4.4). 
Comparing the unsteady and quasi-steady results of two-dimensional simulations, it can be found that quasi-steady simulation self-start Mach number is between 4.3 and 4.4, and two-dimensional unsteady simulation proves its correctness with self-starting Mach number of about 4.36. Both total pressure recovery coefficient and mass flow rate are consistent. For aerodynamic forces, inlet drag and lift of unsteady simulations in Mach 3.9 to Mach 4.3 have the same trend as quasi-steady simulation, but in the range of Mach 4.3 to 4.5 , unsteady simulations obtain drag and lift periodically changes with period of about $0.4 \mathrm{~s}$, because the flow structure of the first cavity after isolator changes between "opened" and "closed" as shown in Figure 24. One time step of Mach number of unsteady simulation is about 0.05 and the Mach number interval of quasi-steady simulation is 0.1 which is just two unsteady time steps of Mach number. This can explain why twodimensional quasi-steady simulation did not get periodic aerodynamic forces changes.

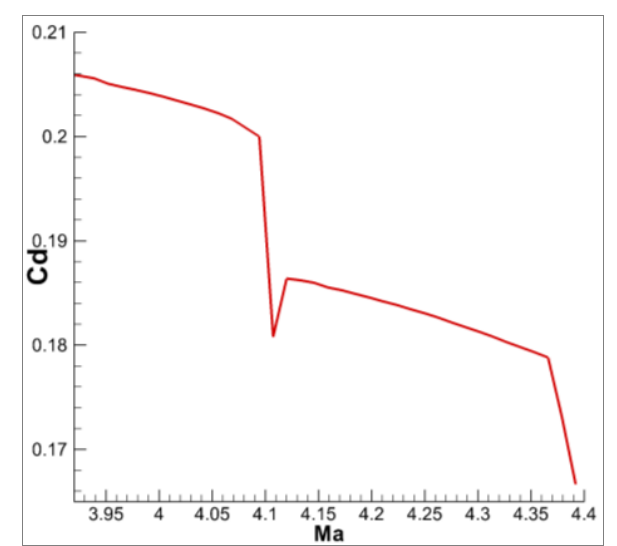

Fig. 20. Drag changes of unsteady simulation (Mach3.9 to 4.4).

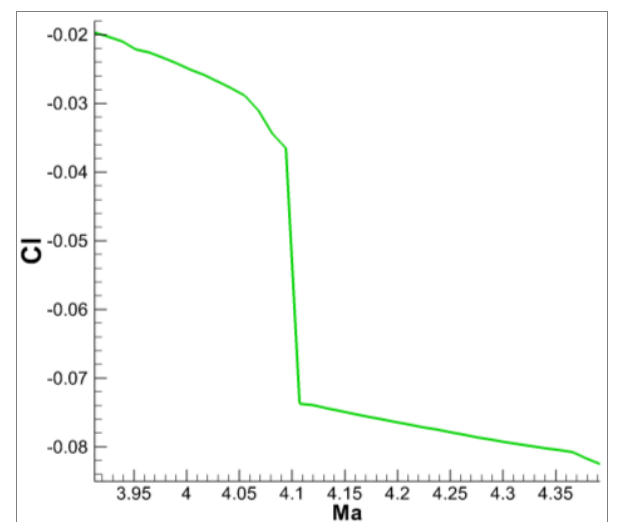

Fig. 21. Lift changes of unsteady simulation (Mach3.9 to 4.4).

\section{ConClusion}

This paper studies the self-starting process of a hypersonic inlet during the vehicle accelerating trajectory with variable free stream condition. Twodimensional quasi-steady and unsteady simulations and three-dimensional quasi-steady simulation are used to analyze the flow phenomena of inlet transiting from unstart to start. We can draw the following conclusions.

1. Self-starting Mach number of the inlet obtained from two-dimensional quasi-steady simulation is between 4.3 and 4.4, and unsteady simulation verified the correctness. The start Mach number of two-dimensional quasi-steady simulation is 4.36 and the three-dimensional result is between 4.0 and 4.1 .

2. There are two sudden changes of aerodynamic forces, pressure, total pressure recovery coefficient and mass flow rate when inlet changes from unstart to start in two-dimensional simulations, but only one jump for threedimensional simulation.

3. The sudden change of flow field parameters is due to changes in the structure of the shock wave. Lip detached shock wave is sucked in the inlet convergent section to make the flow spillage smaller and separation bubble smaller until it is disappeared.

4. Aerodynamic forces of two dimensional unsteady simulation above Mach 4.3 are found to change periodically with a period of about $0.4 \mathrm{~s}$, but it is not found in the corresponding two-dimensional quasi-steady simulation. Aerodynamic analysis indicates that it is due to the flow structure of the first cavity after isolator changes between "opened" and "closed". The quasi-steady simulation can't capture this phenomena because of Mach number interval of calculation is twice larger than unsteady time step.

5. Due to the consistency between the quasi-steady and unsteady in self-starting Mach number in our strategy, for the self-starting process of hypersonic inlet with variable free stream condition, three-dimensional quasi-steady method can be used to approximate the real selfstarting process during the acceleration of the aircraft climb, which can greatly save the computational time and improve simulation efficiency.

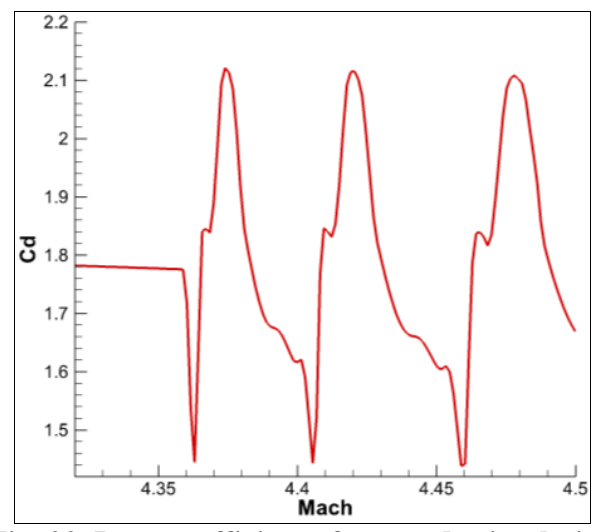

Fig. 22. Drag coefficient of unsteady simulation (Mach4.3 to 4.5). 


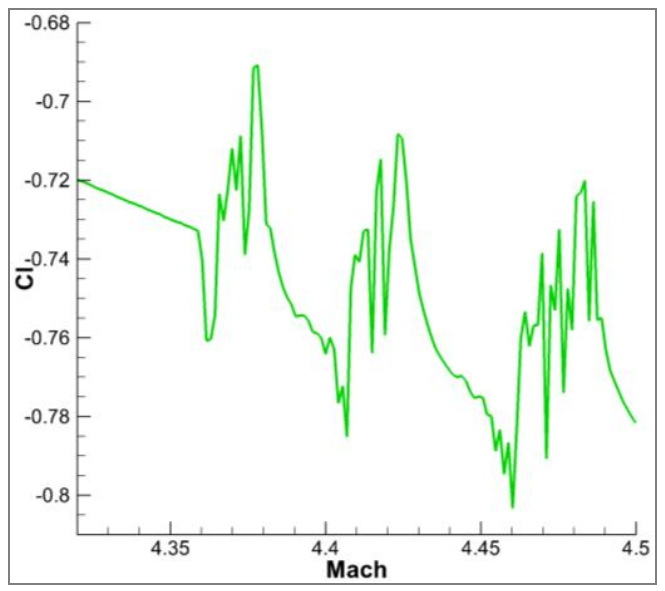

Fig 23. Lift coefficient of unsteady simulation (Mach4.3 to 4.5).

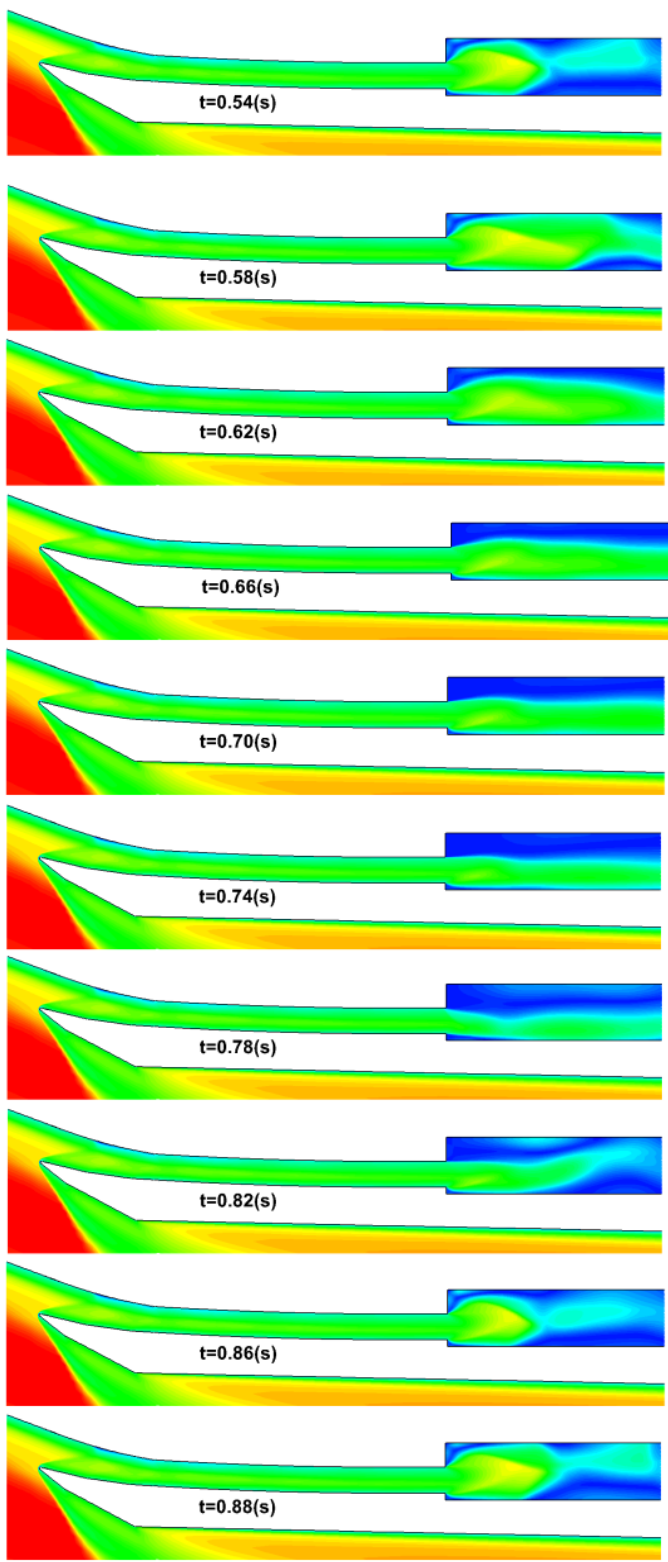

Fig. 24. Mach number contours of cavity during a cycle of period of unsteady simulation.

\section{ACKNOWLEDGEMENTS}

We are grateful for the valuable comments and suggestions made by the reviewers and my colleagues, which are significant contributions in improving the quality of this paper. Jie $\mathrm{Wu}$ was partially supported by the National Natural Science Foundation of China (No.91641107).

\section{REFERENCES}

Chang, J. T, D. R. Yu, W. Bao and L. Qu (2008). Influence Factors of Unstart Boundary for Hypersonic Inlets. 44th AIAA /ASME /SAE /ASEE Joint Propulsion Conference \& Exhibit, 21-23 July 2008, Hartford, CT, AIAA 20084586.

Grainger, A. L., P. R. Boyce, S. C. Tirtey, H. Qgawa, G. Paniagua and S. Paris (2012). The Unsteady Flow Physics of Hypersonic Inlet Starting Processes, $18^{\text {th }}$ AIAA/3AF International Space Planes and Hypersonic Systems and Technologies Conference 24-28 September 2012, Tours, France, AIAA 20125937.

Heiser, W. H., U. Mehta and D. T. Pratt (1994) Hypersonic Airbreathing Propulsion, AIAA Educational Series, AIAA Press, Washington D.C., 1994.

Hsieh, T., A. B. Wardlaw Jr and P. Collins (1984, January). Numerical Investigation of Unsteady Inlet Flow Fields. AIAA 22 $2^{\text {nd }}$ Aerospace Sciences Meeting, January 9-12, 1984, Reno, Nevada, AIAA 84-0031.

Jameson, A. (1991). Time Dependent Calculations Using Multigrid, with Applications to Unsteady Flows Past Airfoils and Wings. $10^{\text {th }}$ Computational Fluid Dynamics Conference, Honolulu HI. AIAA 1991-1596.

Jiao, X. L., J. T. Chang, Z. Q. Wang and D. R. Yu (2015). Mechanism Study on Local Unstart of Hypersonic Inlet at High Mach Number. AIAA Journal 53(10).

Kim, K. H., C. Kim and O. H. Rho (2001). Methods for the Accurate Computations of Hypersonic Flows I.AUSMPW+ Scheme. Journal of Computational Physics 174,38-80.

Kopasakis, G. and J. W. Connolly (2012 July). Quasi One-Dimensional Unsteady Modeling of External Compression Supersonic Inlets. $48^{\text {th }}$ AIAA/ASME/SAE/ASEE Joint Propulsion Conference \& Exhibit 30 July-01 August 2012, Atlanta, Georgia, AIAA 2012-4147.

Leer, B. V. (1979). Towards the Ultimate Conservative Difference Scheme V. A SecondOrder Sequel to Godunov's Method. Journal of Computational Physics 32,101-120.

Logan, P. R., D. V. Gaitonde, M. A. Hagenmaier and J. M. Donbar (2018). Isolator Dynamics During Unstart of a Dual-Mode Scramjet. 
J. Wu et al. / JAFM, Vol. 13, No. 6, pp. 1683-1691, 2020.

Journal of Propulsion and Power 34, (6).

Mayer, D. W. and G. C. Paynter (1994, January). Prediction of Supersonic Inlet Unstart Caused by Freestream Disturbances $32^{\text {nd }}$ Aerospace Sciences Meeting \& Exhibit, January 10-13, 1994, Reno, Nevada, AIAA 94-0580.

McClinton, C. R., J. L. Hunt, R. H. Ricketts, P. Reukauf and C., L. Peddie (1999, November). Airbreathing Hypersonic Technology Vision Vehicles and Development Dreams. 9th International Space Planes and Hypersonic Systems and Technologies conference and 3rd Weakly Ionized Gases Workshop, Norfolk, VA, 1999-4987.

Menter, F. R. (1994). Two-Equation Eddy-Viscosity Turbulence Models for Engineering Applications. AIAA Journal 32(8).

Sebastian, K., J. Matrinez, S. Stuart Laurence and K. Hannemann (2011, April). CFD analysis of Unstart Characteristics of the HyShot II Scramjet Configuration in the HEG Shock Tunnel. 17th AIAA International Space Planes and Hypersonic Systems and Technologies Conference, San Francisco, California, AIAA 2011-2309.

Yoon, S. and A. Jameson (1988). Lower-Upper Symmetric-Gauss-Seidel Method for the Euler and Navier-Stokes Equations. AIAA Journal 26(9), 1025-2016.

Tahhir, R. B., S. Molder and E. V. Timofeev (2003, July). Unsteady Starting of High Mach Number Air Inlets-A CFD study. 39 ${ }^{\text {th }}$ AIAA /ASME ISAE /ASEE Joint Porpulsion Conference and Exhibit. Huntsville, Alabama, AIAA 2003-5191.

Tan, H. J. and R. W. Guo (2007). Experimental Study of the Unstable-Unstarted Condtion of a Hypersonic Inlet at Mach 6. Journal of Propulsion and Power 23(4), 783-788.

Wu, J., Y. Y. He, G. H. Ding and Y. Y. Han (2018). Hybrid Optimized Low-Dissipation and Adaptive MUSCL Reconstruction Technique for Hyperbolic Conservation. Journal of Scientific Computing 77, 552-578. 